\title{
Individual, community and region level predictors of insecticide-treated net use among women in Uganda: a multilevel analysis
}

Edward Kwabena Ameyaw ${ }^{1}$, Yusuf Olushola Kareem ${ }^{2}$ and Sanni Yaya ${ }^{3,4^{*}}$ (D)

\begin{abstract}
Background: Use of insecticide-treated net (ITN) has been identified by the World Health Organization as an effective approach for malaria prevention. The government of Uganda has instituted measures to enhance ITN supply over the past decade, however, the country ranks third towards the global malaria burden. As a result, this study investigated how individual, community and region level factors affect ITN use among women of reproductive age in Uganda.
\end{abstract}

Methods: The 2018-2019 Malaria Indicator Survey of Uganda involving 7798 women aged 15-49 was utilized. The descriptive summaries of ITN use were analysed by individual, community and region level factors. Based on the hierarchical nature of the data, four distinct binomial multilevel logistic regression models were fitted using the MLwiN 3.05 module in Stata. The parameters were estimated using the Markov Chain Monte Carlo (MCMC) estimation procedure and Bayesian Deviance Information Criterion was used to identify the model with a better fit.

Results: The proportion of women who use ITN was $78.2 \%(n=6097)$. Poor household wealth status [aOR $=1.66$, $\mathrm{Crl}=1.55-1.80]$, knowing that sleeping under ITN prevents malaria [aOR $=1.11, \mathrm{Crl}=1.05-1.24]$ and that destroying mosquito breeding sites can prevent malaria $[\mathrm{aOR}=1.85, \mathrm{Crl}=1.75-1.98]$ were associated with higher odds of ITN use. ITN use attributable to regional and community level random effects was $39.1 \%$ and $45.2 \%$, respectively.

Conclusion: The study has illustrated that ITN policies and interventions in Uganda need to be sensitive to community and region level factors that affect usage. Also, strategies to enhance women's knowledge on malaria prevention is indispensable in improving ITN use.

Keywords: Insecticide-treated net, ITN, Women, Malaria, Uganda, Public health, Global health, Malaria Indicator Survey

\section{Background}

Malaria among women in the reproductive age has become a public health priority due to the enormous adverse implications it poses to their reproductive wellbeing [1]. Six countries account for more than half of the global malaria burden. All these countries are located in

\footnotetext{
*Correspondence: sanni.yaya@uOttawa.ca

${ }^{3}$ School of International Development and Global Studies, University of Ottawa, Ottawa, Canada

Full list of author information is available at the end of the article
}

sub-Saharan Africa and includes Uganda where $5 \%$ of the global malaria cases occur [2]. Malaria is a public health priority linked with poverty, declined socio-economic development and is the commonly reported disease within both public and private health facilities in Uganda [3]. It is endemic in $95 \%$ of Uganda with the remaining $5 \%$ being irregular and epidemic-prone transmission areas [4]. Uganda accounts for the sixth highest annual malaria induced deaths in Africa with $\$ 0.41$ to $\$ 3.88$ malaria expenditure per person per month. One episode of malaria costs a family an average of $3 \%$ of their annual

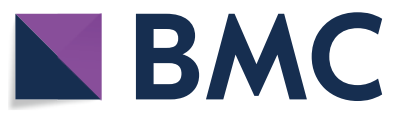

(c) The Author(s) 2020. This article is licensed under a Creative Commons Attribution 4.0 International License, which permits use, sharing, adaptation, distribution and reproduction in any medium or format, as long as you give appropriate credit to the original author(s) and the source, provide a link to the Creative Commons licence, and indicate if changes were made. The images or other third party material in this article are included in the article's Creative Commons licence, unless indicated otherwise in a credit line to the material. If material is not included in the article's Creative Commons licence and your intended use is not permitted by statutory regulation or exceeds the permitted use, you will need to obtain permission directly from the copyright holder. To view a copy of this licence, visit http://creativeco mmons.org/licenses/by/4.0/. The Creative Commons Public Domain Dedication waiver (http://creativecommons.org/publicdomain/ zero/1.0/) applies to the data made available in this article, unless otherwise stated in a credit line to the data. 
revenue. These include direct out-of-pocket expenses on consultation, drugs and transportation cost to distant health facilities [4].

Insecticide-treated net (ITN) has been identified by the World Health Organization (WHO) and the global community as an effective approach for malaria prevention. The government of Uganda has acknowledged this and instituted measures to enhance ITN supply to households. In the year 2000, the government waived importation taxes on ITNs and this was followed by establishment of the ITN Working Group of the interagency coordination committee for malaria (ICCM) in 2002. In 2009, the country scaled up to universal coverage of ITN (i.e. one ITN for every two persons). As of 2014, the National Malaria Control Program (NMCP) had distributed more than 12 million Long Lasting Insecticide Nets (LLNs) through mass campaigns and this culminated in $60 \%$ administrative coverage. The prime vision of the NMCP is to achieve "Malaria free Uganda" by providing quality reliable malaria prevention and treatment services to all persons. Women and children constitute the priority group of these interventions due to reduced immunity during pregnancy and immature immune system of children $[1,4]$.

Although, recent studies have revealed high ITN ownership in Uganda. However, ownership of ITN do not commensurate with utilisation. Nuwamanya et al. [5] reported $98.8 \%$ ownership of ITN with $91.1 \%$ of utilization. Although, this study was conducted after a mass ITN distribution exercise in Mbarara municipality. Similarly, another study in south western Uganda reported $84.0 \%$ of ownership of ITN among pregnant women, and only $66.1 \%$ reported consistent use [6]. In spite of the gap between ITN ownership and usage [5-7], little is known about the individual and contextual level factors associated with ITN use among women of reproductive age. Previously reported association between socio-demographics and ITN use have also been inconsistent in the literature. For example, a significantly positive association has been found between older age [8], higher education [9], and better household wealth [10] and ITN use, whereas some studies have shown that the association were insignificant or otherwise [11]. This study investigates individual, community and regional level factors associated with ITN use among women of reproductive age in Uganda.

\section{Methods}

\section{Study design}

The 2018-2019 Malaria Indicator Survey of Uganda (2018-19 UMIS) was utilized for this study. UMIS is a cross-sectional survey implemented by the Uganda
Bureau of Statistics (UBOS) and National Malaria Control Division (NMCD) with technical support from the Inner City Fund (ICF) [12].

\section{Sampling technique}

A two-stage sampling design was adopted in order to ensure the estimation of core indicators for three cardinal domains; national, rural/urban settings and the 15 regions of the country. Refugee settlements were captured as a distinct sampling domain. In the first phase of sampling, sample points (clusters) were selected from the sample frames, that is refugee and non-refugee settlements. The Enumeration Areas (EAs) earmarked for the 2014 National Population and Housing Census (NPHC) were used as the sampling frame. Three hundred and twenty (320) clusters were selected from the non-refugee settlement frame through probability proportional to size as defined by the NPHC. This emerged from rural (236) and urban (84) areas of the country. Within regions, urban settings were oversampled in order to generate unbiased estimations for urban domains. For refugee frame, 22 clusters were selected following the same procedure. The second phase constituted a systematic selection of households by listing households and eligible respondents were randomly selected thereafter. A total of 28 households were selected from each EA yielding a total sample size of 8878 households. All women aged 15-49 who were either permanent residents of selected households or visitors who joined the households the night before the survey were eligible for the survey. Within selected households, 8389 women were eligible for interview and 8231 were interviewed successfully culminating in $98 \%$ response rate for the non-refugee settlements while the refugee settlement, had $99 \%$ response rate [12].

\section{Inclusion criteria and sample size}

This study included women aged 15-49 who indicated that they possessed ITN during the 2018-2019 Malaria Indicator survey. Based on this, a total of 7798 women were included in the analysis.

\section{Data collection}

The data collection exercise occurred between December 11, 2018 and January 31, 2019. Twenty-three teams were formed for the data collection and each of these had seven members. Information about these women were gathered with three questionnaires. These are the household questionnaire, woman's questionnaire and the biomarker questionnaire. To mitigate possible language barrier and ensure clarity, the questionnaires were translated from English Language to the local languages namely Luganda, Luo, Lugbara, Ateso, Runyankole/ 
Rukiga, and Runyoro/Rutoro. Whilst the household and woman's questionnaires were programmed to tablets to permit computer-assisted personal interviewing (CAPI), the biomarker questionnaire was in hard copy and subsequently entered to the CAPI platform after completion. Listing of usual members of households and visitors, gathering of basic information on each listed person and data on characteristics of the households' dwelling unit were done with the household questionnaire. The basic information comprised age, sex and relationship to the household head. Characteristics of household dwelling unit included toilet facilities, indoor residual spraying, and ownership and use of mosquito nets. The woman's questionnaire was used for collecting information from all women aged 15-49 whilst the biomarker questionnaire was used to capture the results of the anaemia and malaria testing of the children 0-59 months.

\section{Outcome variable}

ITN use was the outcome variable for the study. Those who indicated that they slept under the treated mosquito net the night before the survey were considered as using ITN (coded as 1) whereas those who did not sleep under net were considered as not using ITN (coded as 0 ).

\section{Explanatory variables \\ Individual level factors}

Eight variables were considered at the individual level; age, education, household wealth index, pregnancy status, if mosquito bite causes malaria, if sleeping under ITN prevents malaria, if destroying mosquito breeding sites prevent malaria and household size. Age was categorized as $15-19,20-24,25-29,30-34,35-39,40-44$ and $45-49$. Education was measured as highest level of educational attainment and categorized into no education, primary, secondary and higher. The household wealth index was derived from aggregation of household's asset possession. Thus, items such as type of floor/roofing, radio, television, car, toilet and source of water were computed into wealth index through principal component analysis and categorized into poorest, poorer, middle, richer and richest. These were re-categorized into poor middle and rich. Pregnancy status was classified as pregnant, no or not sure. Household size was categorized into less than five and five or more. These three variables: if mosquito bite causes malaria, if sleeping under ITN prevents malaria and if destroying mosquito breeding sites prevents malaria, had binary responses (yes or no).

\section{Community level factors}

Three variables were considered at the community level. The first was residence categorized as urban, rural and refugee settlements. The second variable was socio-economic status computed using principal component analysis and comprised proportion of women who were poor and uneducated within communities. The socioeconomic status was categorized into tertiles: tertile 1 (least disadvantaged), tertile 2 and tertile 3 (most disadvantaged). The third variable was region categorized into Eastern, Northern, Western and Southern Uganda.

\section{Region level factors}

Socio-economic status at the regional level was derived by using principal component analysis to compute the proportion of women who were poor and uneducated within the regions and then categorized into tertiles: tertile 1 (least disadvantaged), tertile 2 and tertile 3 (most disadvantaged).

\section{Statistical analysis}

The descriptive summaries of the individual, community and regional level factors by ITN utilization were computed. A Chi squared test of association was performed for each of the explanatory variable and ITN use. Four distinct multilevel binary logistic regression models with hierarchical structure were fitted thereafter. The first (null) model had no explanatory variable. The null model decomposed the magnitude of variation in ITN use that can be explained by community and regional random effects. The second model then included the individuallevel variables, the third model comprised both individual and community level variables while the fourth (full) model, controlled for individual, community and region level factors. The fixed effects of all the models were computed using odds ratios (ORs) with corresponding 95\% credible intervals (Crls) whereas the random effects were measured with intra-cluster correlation coefficient (ICC) and median odds ratio (MOR) $[13,14]$.

\section{Model fit and specification}

Multicollinearity was checked using variance inflation factor (VIF) with greater than 5 as cut-off [15]. The parameters were estimated using the Markov Chain Monte Carlo (MCMC) [16] approach. In order to fit the most parsimonious model, the Bayesian Deviance Information Criterion (DIC) was used as a test for model fit, a model with a lower value of DIC has a better fit. All the fitted models were analysed using the MLwiN command version 3.05 [17] in the Stata software version 13 (StataCorp, College Station, TX).

\section{Ethics approval}

Protocol for the 2018-2019 UMIS was approved by the Uganda National Council for Science and Technology (UNCST), the Ethics Committee of the School of Medicine Research and Ethics Committee (SOMREC) 
of the Makerere University and the institutional review board at the ICF. All participants consented before participating in the survey and the data were anonymized at the data finalization stage.

\section{Results \\ Descriptive results}

Table 1 presents ITN use among Ugandan women by socio-demographic characteristics at the individual, community and region level. A total of 6097 women (78.2\%) used ITN. A higher proportion of women age 45-49 (85.8\%) and with tertiary education (83.4\%) utilized ITN. Most women who knew that mosquito bite causes malaria reported ITN use (79.6\%). A higher proportion of those who indicated that sleeping under mosquito net prevents malaria utilized ITN (78.6\%) and similarly among women who indicated that destroying mosquito breeding sites can prevent malaria (79.5\%). Most women in households with 5 or more members indicated that they utilized ITN $(85.1 \%)$. At the community level, a higher proportion of women $(88.1 \%)$ who resided in refugee settlements, most disadvantaged communities $(79.8 \%)$ and in Southern Uganda (81.3\%) reported ITN use. At the regional level, a higher proportion of women in a moderately disadvantaged region $(78.6 \%)$ utilized ITN compared to women in the least and most disadvantage regions.

\section{Measures of association (fixed effects)}

The results for the fitted models were presented in Table 2. The multicollinearity test indicated that there was no collinearity between the independent variables [highest VIF $=3.71$, lowest $\mathrm{VIF}=1.72$, mean VIF $=2.08]$. The final model consists of individual, community and region level variables. Household size of five or more, poor household wealth status, knowledge on whether sleeping under mosquito net prevents malaria and whether destroying mosquitobreeding site can prevent malaria were positively associated with ITN use. Women in poor household wealth quintile $[\mathrm{aOR}=1.66, \mathrm{Crl}=1.55-1.80]$ had higher odds of ITN utilisation compared to those in the rich quintile. Women who perceive that sleeping under ITN prevents malaria had a higher odds of ITN use $[\mathrm{aOR}=1.11, \mathrm{Crl}=1.05-1.24]$. Similarly, women who believed that destroying mosquito breeding sites can prevent malaria had higher odds of ITN use compared to those who did not think so $[\mathrm{aOR}=1.85$, $\mathrm{Crl}=1.75-1.98]$. Women whose household members were less than five were associated with lower odds of ITN use $[\mathrm{aOR}=0.66, \mathrm{Crl}=0.58-0.74]$. Also, rural residents were less likely to use ITN $[\mathrm{aOR}=0.63$, $\mathrm{Crl}=0.41-0.98]$.

\section{Measures of variation (random effects)}

The results of the random effects are also presented in Table 2. Outcome of the first model (empty) indicated that substantial variation in ITN use exist across regions $\left[\sigma^{2}=2.34,95 \% \mathrm{Crl}=1.87-2.94\right]$. The intra-region correlation coefficient shows that $39.10 \%[\mathrm{Crl}=35.7-44.2]$ of the variance in the likelihood of ITN use is attributable to region level factors. Also, $45.2 \%$ odds $[\mathrm{Crl}=37.0-50.4]$ of ITN use is attributable to community level factors. From model 4, the MOR reveals that when a woman relocates to a different region with higher likelihood of ITN use, the odds of ITN utilisation increases by 4.28 . Similarly, the odds of ITN utilization increases by 3.57 if a woman moves to a community with a higher likelihood of ITN use.

\section{Discussion}

This study aimed at investigating how individual, community and region level factors affect ITN use among women aged 15-49 in Uganda. The findings indicate that indeed factors at these three levels affect ITN use in the country. This suggests that ITN use transcends personal level characteristics and highlights the importance of community and region level factors. It therefore calls for a hierarchical intervention that considers the dynamics of ITN use and malaria prevalence at the regional, community and household levels. Previous studies have indicated discomforts such as heat, scent, net washing, vomiting and difficulty in hanging the net among other factors interfere usage [18-21]. In the case of Uganda, this current study draws attention to the need to investigate which of these arrays of factors attenuate ITN use in the respective communities and regions so as to revise current interventions accordingly to render them more relevant and responsive.

Poor women were more likely to use ITN. This outcome resonates with evidence from Rwanda and Ghana [22, 23]. Through the National Malaria Control Programme, the President's Malaria Initiative and other anti-malaria interventions, the government of Uganda and other stakeholders have ensured easy access to ITN and other malaria control measures [3, 4]. For instance, over 7.2 million LLINs were distributed in 2010 under the National Malaria Control Programme and as of 2013/2014, about 12 million LLINs had been distributed through mass campaigns where $60 \%$ administrative coverage was achieved with the aim of achieving the "Malaria free Uganda" vision [4]. These interventions 
Table 1 ITN use at individual, community and region level independent variables. (Source: 2018-19 Uganda Malaria Indicator Survey)

\begin{tabular}{|c|c|c|c|c|}
\hline & \multicolumn{3}{|c|}{ ITN UTILISATION } & \multirow[t]{3}{*}{$p$ value } \\
\hline & No & Yes & Total & \\
\hline & n (\%) & n (\%) & n (\%) & \\
\hline \multicolumn{5}{|l|}{ Individual level } \\
\hline Age & & & & $<0.001$ \\
\hline 15-19 & $600(33.4)$ & $1196(66.6)$ & $1796(100)$ & \\
\hline $20-24$ & $329(22.1)$ & $1153(77.8)$ & $1481(100)$ & \\
\hline $25-29$ & $244(18.9)$ & $1048(81.1)$ & $1292(100)$ & \\
\hline $30-34$ & $185(16.5)$ & $934(83.5)$ & $1119(100)$ & \\
\hline $35-39$ & $147(16.3)$ & $755(83.7)$ & $902(100)$ & \\
\hline $40-44$ & $128(17.7)$ & $595(78.3)$ & $724(100)$ & \\
\hline $45-49$ & $69(14.2)$ & $414(85.8)$ & $483(100)$ & \\
\hline Education & & & & $<0.01$ \\
\hline No education & $191(19.1)$ & 806 (80.9) & $997(100)$ & \\
\hline Primary & $920(22.7)$ & $3131(77.3)$ & $4051(100)$ & \\
\hline Secondary & $501(22.6)$ & $1712(77.4)$ & $2213(100)$ & \\
\hline Higher & $89(16.6)$ & 448 (83.4) & $537(100)$ & \\
\hline Household wealth index & & & & $<0.01$ \\
\hline Poor & $400(21.3)$ & $1478(78.7)$ & $1878(100)$ & \\
\hline Middle & $621(22.9)$ & $2087(77.1)$ & $2709(100)$ & \\
\hline Rich & $680(21.2)$ & $2531(74.0)$ & $3211(100)$ & \\
\hline Pregnancy status & & & & $<0.01$ \\
\hline No or not sure & $1569(21.8)$ & $5616(78.2)$ & $7185(100)$ & \\
\hline Pregnant & $132(21.5)$ & $481(78.5)$ & $613(100)$ & \\
\hline Mosquito bite causes malaria & & & & 0.065 \\
\hline No & $1124(22.6)$ & $3850(77.4)$ & $4974(100)$ & \\
\hline Yes & $577(20.4)$ & $2247(79.6)$ & $2824(100)$ & \\
\hline Sleeping under mosquito net prevents malaria & & & & $<0.001$ \\
\hline No & $443(23.0)$ & $1486(77.0)$ & $1929(100)$ & \\
\hline Yes & $1258(21.4)$ & $4611(78.6)$ & $5869(100)$ & \\
\hline Destroying mosquito breeding site can prevent malaria & & & & 0.828 \\
\hline No & $1369(22.2)$ & $4804(77.8)$ & $6173(100)$ & \\
\hline Yes & $332(20.5)$ & $1292(79.5)$ & $1625(100)$ & \\
\hline Household size & & & & $<0.001$ \\
\hline Less than 5 & $1391(24.4)$ & $4321(75.6)$ & $5712(100)$ & \\
\hline 5 or more & $310(14.9)$ & $1775(85.1)$ & $2085(100)$ & \\
\hline \multicolumn{5}{|l|}{ Community level factors } \\
\hline Residence & & & & $<0.001$ \\
\hline Urban & $410(19.2)$ & $1724(80.8)$ & $2135(100)$ & \\
\hline Rural & $1229(23.9)$ & $3914(76.1)$ & $5143(100)$ & \\
\hline Refugee settlements & $62(11.9)$ & $458(88.1)$ & $520(100)$ & \\
\hline Socio-economic disadvantage & & & & $<0.01$ \\
\hline Tertile 1 (least disadvantaged) & $655(20.9)$ & $2472(79.1)$ & $3127(100)$ & \\
\hline Tertile 2 & $692(23.8)$ & $2222(76.2)$ & $2914(100)$ & \\
\hline Tertile 3 (most disadvantaged) & $354(20.2)$ & $1403(79.8)$ & $1757(100)$ & \\
\hline Region & & & & $<0.001$ \\
\hline Eastern & $642(24.2)$ & 2005 (75.8) & $2647(100)$ & \\
\hline Northern & $415(22.5)$ & $1431(77.5)$ & $1846(100)$ & \\
\hline Western & $298(20.4)$ & $1162(79.6)$ & $1460(100)$ & \\
\hline
\end{tabular}




\begin{tabular}{|c|c|c|c|c|}
\hline & \multicolumn{3}{|c|}{ ITN UTILISATION } & \multirow[t]{3}{*}{$p$ value } \\
\hline & No & Yes & Total & \\
\hline & n (\%) & n (\%) & n (\%) & \\
\hline Southern & $346(18.7)$ & $1499(81.3)$ & $1845(100)$ & \\
\hline Region level factors & & & & $<0.001$ \\
\hline \multicolumn{5}{|l|}{ Socio-economic disadvantage } \\
\hline Tertile 1 (least disadvantage) & $947(23.0)$ & $3164(77.0)$ & $411(100)$ & \\
\hline Tertile 2 & $420(20.0)$ & $1707(80.0)$ & $2127(100)$ & \\
\hline Tertile 3 (most disadvantaged) & $334(21.4)$ & $1226(78.6)$ & $1560(100)$ & \\
\hline $\mathrm{N}$ & $1701(21.8)$ & 6097 (78.2) & 7798 (100) & \\
\hline
\end{tabular}

are predominantly pro-poor because the rich are more likely to reside in less malaria prone environments unlike the poor who may be in environments with poor drainage system and unkempt environments where mosquito breeding sites are immanent $[22,24]$. On the account of these, the poor are likely to embrace and effectively use ITNs. Due to the economic advantageous position of the rich, they can explore other malaria prevention strategies such as indoor residual spraying (IRS) or use of mosquito repellents with ease [24, 25]. Conversely, these options may be "luxurious" to the poor women and hence effective utilisation of readily available ITN. The finding indicates that ITN distribution, advocacy interventions and policies need to target poor women in Uganda because they may have a critical demand for ITNs relative to the rich.

Women who perceived that sleeping under ITN prevents malaria were more likely to sleep under ITN. Similarly, women with the perception that destroying mosquito breeding site can prevent malaria had higher odds of using ITN. Undoubtedly, women who know these preventive strategies are more knowledgeable about the causes of malaria and its prevention [26]. These findings reflect the proposition of the health belief model [27]. Strecher and Rosenstock [27] espoused that one's ability to initiative a healthy or protective intervention or lifestyle is not only a function of the person's characteristics but also the wealth of knowledge and information held about the phenomenon. On this score, high ITN use is expected among women who believe that sleeping under ITN and destroying mosquito-breeding sites can prevent malaria. Our observed results concur with reports from South Eastern Asia [18, 28, 29]. The findings prompt a compelling need to intensity ITN education and mass media campaigns on the importance of ITN use and risks associated with doing otherwise in the absence of other preventive measures. Such education and campaigns can have wider coverage and higher impact when channelled through widely utilized mass media platforms such as radio, which is noted to be the most vibrant and commonest medium for public discussion and ITN advertisements in Uganda [30, 31].

Further, women in households with less than five members were less likely to use ITN. This finding in inconsistent with the observation made by Nkoka et al. [32] in Malawi where ITN use was more probable for women from households with less persons. This finding is, however, consistent with a Cameroon-based study on coverage and usage of insecticide-treated nets (ITNs) within households. Unlike smaller households, households with greater or more members are more likely to have someone who can educate other household members on the relevance of ITN [33].

Women in rural locations also had less likelihood of ITN use. This may reflect inequity in ITN outlets in favour of urban locations or limited education in rural settings. In contrast, Bennett et al. [34] noted that rural residents in Cameroon stand a higher chance of using ITN compared with urban residents following a national mass distribution exercise. Another study from Ghana revealed that rural residents are more likely to use ITN [22]. Contextual variations between Uganda and settings for the other studies may account for the inconsistent findings. Scrutiny of rural/urban distribution of ITN and advocacy strategies may be required to enhance ITN use in rural Uganda.

\section{Strengths and limitations}

This study is supported by data from the most recent malaria survey of Uganda and, therefore, presents current estimates. The study uses large sample size, which renders the findings generalizable to Uganda women. The study offers information on ITN use taking cognisance of community and region level factors. In spite of these strengths, the study is not devoid of limitations. First, 
Table 2 Multilevel logistic regression of individual, community and region level correlates of ITN use (Source: 2018-19 Uganda Malaria Indicator Survey)

Model 1

Model 2

OR [95\% Crl]

Fixed effect

Individual level

Age

$15-19$

20-24

$25-29$

30-34

35-39

40-44

45-49

Education

No education

Primary

Secondary

Higher

Household wealth index

Poor

Middle

Rich

Pregnancy status

No or not sure

Pregnant

Mosquito bite causes malaria

No

Yes

Sleeping under mosquito net prevents malaria

No

Yes

Destroying mosquito breeding site can prevent malaria

No

Yes

Household size

Less than 5

5 or more

Community level factors

Residence

Urban

Rural

Refugee settlements

Socio-economic disadvantage

Tertile 1 (least disadvantaged)

Tertile 2

Tertile 3 (most disadvantaged)

Region

Eastern

Northern

Western

\begin{tabular}{|c|c|c|}
\hline $0.40[0.32-0.50]^{* * *}$ & $0.41[0.33-0.51]^{* *}$ & $0.39[0.31-0.49]^{* * *}$ \\
\hline $0.67[0.54-0.83]^{* * *}$ & $0.70[0.56-0.87]^{* *}$ & $0.66[0.51-0.84]^{* * *}$ \\
\hline 0.87[0.69-1.09] & $0.90[0.72-1.13]$ & $0.86[0.67-1.10]$ \\
\hline $1.08[0.86-1.36]$ & $1.13[0.90-1.42]$ & $1.07[0.83-1.38]$ \\
\hline $1.07[0.84-1.35]$ & $1.10[0.86-1.41]$ & $1.05[0.80-1.37]$ \\
\hline $0.96[0.74-1.24]$ & $0.99[0.76-1.28]$ & $0.94[0.72-1.23]$ \\
\hline Ref & Ref & Ref \\
\hline $0.70[0.52-0.94]^{* *}$ & $0.77[0.58-1.01]$ & $0.72[0.54-0.96]^{*}$ \\
\hline $0.86[0.66-1.11]$ & $0.93[0.72-1.19]$ & $0.86[0.68-1.10]$ \\
\hline $0.92[0.71-1.18]$ & $0.99[0.78-1.26]$ & $0.93[0.73-1.18]$ \\
\hline Ref & Ref & Ref \\
\hline $1.64[1.54-1.76]^{* * *}$ & $1.65[1.54-1.78]^{* * *}$ & $1.66[1.55-1.80]^{* *}$ \\
\hline $1.83[1.71-1.97]^{* *}$ & $1.83[1.71-1.96]^{* *}$ & $1.84[1.72-1.98]^{* * *}$ \\
\hline Ref & Ref & Ref \\
\hline $0.93[0.78-1.11]$ & $0.97[0.81-1.15]$ & $0.94[0.79-1.12]$ \\
\hline Ref & Ref & Ref \\
\hline $0.90[0.81-1.01]$ & $0.91[0.82-1.01]$ & $0.90[0.81-1.00]$ \\
\hline Ref & Ref & Ref \\
\hline Ref & Ref & Ref \\
\hline $1.12[0.99-1.26]$ & $1.13[1.01-1.26]^{*}$ & $1.11[1.05-1.24]^{* *}$ \\
\hline $0.87[0.76-0.99]^{*}$ & $0.87[0.75-0.99]^{*}$ & $1.85[1.75-1.98]^{*}$ \\
\hline Ref & Ref & Ref \\
\hline $0.66[0.59-0.74]^{* * *}$ & $0.66[0.59-0.75]^{* *}$ & $0.66[0.58-0.74]^{* * *}$ \\
\hline Ref & Ref & Ref \\
\hline 0.87[0.59-1.29] & $0.93[0.55-1.56]$ & $0.72[0.44-1.18]$ \\
\hline $0.76[0.54-1.06]$ & $0.77[0.49-1.22]$ & $0.63[0.41-0.98]^{*}$ \\
\hline \multirow[t]{7}{*}{ Ref } & Ref & Ref \\
\hline & Ref & Ref \\
\hline & $1.04[0.84-1.29]$ & $1.02[0.82-1.26]$ \\
\hline & $0.87[0.66-1.16]$ & $0.89[0.67-1.18]$ \\
\hline & $1.71[0.94-3.11]$ & $1.30[0.70-2.42]$ \\
\hline & $2.20[1.15-4.24]$ & $5.08[1.95-13.20]^{* * *}$ \\
\hline & $2.22[0.99-4.95]$ & $1.35[0.69-2.63]$ \\
\hline
\end{tabular}

Model 4

aOR $[95 \% \mathrm{Crl}]$ 
Table 2 (continued)

\begin{tabular}{|c|c|c|c|c|}
\hline & Model 1 & $\begin{array}{l}\text { Model } 2 \\
\text { OR }[95 \% \mathrm{Crl}]\end{array}$ & $\begin{array}{l}\text { Model } 3 \\
\text { OR [95\% Crl] }\end{array}$ & $\begin{array}{l}\text { Model } 4 \\
\text { aOR }[95 \% \mathrm{Crl}]\end{array}$ \\
\hline Southern & & & Ref & Ref \\
\hline \multicolumn{5}{|l|}{ Region level factors } \\
\hline \multicolumn{5}{|l|}{ Socio-economic disadvantage } \\
\hline Tertile 1 (least disadvantage) & & & & Ref \\
\hline Tertile 2 & & & & $1.26[0.79-1.99]$ \\
\hline Tertile 3 (most disadvantaged) & & & & $0.33[0.14-0.74]^{* * *}$ \\
\hline \multicolumn{5}{|l|}{ Measures of variation } \\
\hline \multicolumn{5}{|l|}{ Region level } \\
\hline Variance (SE) & $2.342[1.869-2.936]$ & $0.168[0.061-0.391]$ & $0.181[0.050-0.469]$ & $0.052[0.003-0.175]$ \\
\hline ICC (\%) & $39.1[35.7-44.2]$ & $32[27.8-36.1]$ & $37.45[32.74-43.27]$ & $46.29[42.13-52.62]$ \\
\hline MOR & $4.30[3.68-5.13]$ & $4.19[3.82-4.53]$ & $3.98[3.22-4.31]$ & $4.28[3.99-4.58]$ \\
\hline Explained variation (\%) & Reference & $52.86[48.41-59.34]$ & 48.72[42.74-54.79] & $68.11[63.41-72.63]$ \\
\hline \multicolumn{5}{|l|}{ Community level } \\
\hline Variance (SE) & $0.349[0.068-0.414]$ & $0.344[0.259-0.448]$ & $0.352[0.269-0.450]$ & $0.352[0.270-0.447]$ \\
\hline ICC (\%) & $45.20[37.0-50.4]$ & $37.62[31.39-42.85]$ & $44.21[38.74-47.31]$ & $48.41[40.55-52.17]$ \\
\hline MOR & $1.76[1.28-1.85]$ & $2.38[2.04-3.02]$ & $3.54[3.12-4.11]$ & $3.57[3.13--4.11]$ \\
\hline Explained variation (\%) & Reference & $41.62[38.43-50.8]$ & $39.51[33.59-43.29]$ & $40.32[37.66-46.23]$ \\
\hline \multicolumn{5}{|l|}{ Model fit statistics } \\
\hline Bayesian DIC & $10,345.46$ & $10,073.41$ & $10,073.12$ & $10,072.31$ \\
\hline $\mathrm{N}$ & 7798 & 7798 & 7798 & 7798 \\
\hline
\end{tabular}

DIC Deviation information criterion, $\mathrm{Cr}$ credible interval, ICC intra-cluster correlation, MOR median odds ratio, SE standard error

${ }^{*} p<0.05,{ }^{* *} p<0.01,{ }^{* * *} p<0.001$

depending on a woman's community, there is the possibility of social desirability bias about ITN use. Secondly, this is a cross-sectional study and as such causal inference in not possible.

\section{Conclusions}

The study has indicated that community and region level factors influence ITN use in Uganda. Being poor and possession of knowledge about malaria prevention increased prospects of ITN use. There is, therefore, a need to review the existing ITN inventions and revise taking cognisance of community, and regional level variations. Moreover, the socio-economic status of women and depth of knowledge ITN are critical factors worth considering in planning. Future study may focus on rural-urban variation in ITN use in Uganda.

\section{Abbreviations}

aOR: Adjusted Odds Ratio; CAPI: Computer-assisted personal interviewing; Crl: Credible interval; DIC: Deviance Information Criterion; EAs: Enumeration areas; ICCM: Inter-agency coordination committee for malaria; ICF: Inner City Fund; ICC: Intra-cluster correlation coefficient; ITN: Insecticide-treated net; LLIINs: Long Lasting Insecticide Nets; MCMC: Markov Chain Monte Carlo; MOR: Median odds ratio; NPHC: National Population and Housing Census; NMCD: National Malaria Control Division; NMCP: National Malaria Control Programme;
SOMREC: School of Medicine Research and Ethics Committee; UMIS: Uganda Malaria Indicator Survey of Uganda; UNCST: Uganda National Council for Science and Technology; VIF: Variance inflation factor; WHO: World Health Organization.

\section{Acknowledgements}

The authors are gratefully thankful to the Measure DHS program for the data.

\section{Authors' contributions}

SY and EKA conceptualized and designed the study, analysed the data and drafted the manuscript. YOK provided technical support in interpretation of results and critically reviewed the manuscript for its intellectual content. SY had final responsibility to submit. All authors read and revised drafts of the paper and approved the final version. All authors read and approved the final manuscript.

\section{Funding}

No funding was received for this study.

\section{Availability of data and materials}

The dataset supporting the conclusions of this article is available in the Measure DHS repository, [https://www.malariasurveys.org].

Ethics approval and consent to participate

Not applicable.

\section{Consent for publication}

Not applicable.

\section{Competing interests}

The authors declare that they have no competing interests. 


\section{Author details}

${ }^{1}$ School of Public Health, Faculty of Health, University of Technology Sydney, Sydney, NSW, Australia. ${ }^{2}$ Institute for Advanced Medical Research and Training, College of Medicine, University of Ibadan, Ibadan, Nigeria. ${ }^{3}$ School of International Development and Global Studies, University of Ottawa, Ottawa, Canada.

${ }^{4}$ The George Institute for Global Health, Imperial College London, London, UK.

Received: 17 June 2020 Accepted: 7 September 2020

Published online: 16 September 2020

\section{References}

1. WHO. Malaria in pregnant women. Geneva: World Health Organization; 2017. https://www.who.int/malaria/areas/high_risk_groups/pregnancy/ en/. Accessed 6 June 2020.

2. WHO. World malaria report 2019. Geneva: World Health Organization; 2019.

3. Ministry of Health. Uganda national malaria control policy. Ministry of Health: Republic of Uganda; 2011.

4. Goverment of Uganda. President's Malaria Initiative: malaria operational plan FY 2019. Uganda; 2019.

5. Nuwamanya S, Kansiime N, Aheebwe E, Akatukwasa C, Nabulo H, Turyakira $\mathrm{E}$, et al. Utilization of Long-lasting insecticide treated nets and parasitaemia at 6 months after a mass distribution exercise among households in Mbarara Municipality, Uganda: a cross-sectional community based study. Malar Res Treat. 2018;2018:4387506.

6. Taremwa IM, Ashaba S, Adrama HO, Ayebazibwe C, Omoding D, Kemeza I, et al. Knowledge, attitude and behaviour towards the use of insecticide treated mosquito nets among pregnant women and children in rural Southwestern Uganda. BMC Public Health. 2017;17:794.

7. Ahmed SM, Zerihun A. Possession and usage of insecticidal bed nets among the people of Uganda: is BRAC Uganda Health Programme pursuing a pro-poor path? PLOS ONE. 2010;5:e12660.

8. Aluko JO, Oluwatosin AO. Utilization of insecticide treated nets during pregnancy among postpartum women in Ibadan, Nigeria: a cross-sectional study. BMC Pregnancy Childbirth. 2012;12:21.

9. Muhumuza E, Namuhani N, Balugaba BE, Namata J, Kiracho EE. Factors associated with use of malaria control interventions by pregnant women in Buwunga subcounty, Bugiri District. Malar J. 2016;15:342.

10. Choonara S, Odimegwu CO, Elwange BC. Factors influencing the usage of different types of malaria prevention methods during pregnancy in Kenya. Afr Health Sci. 2015;15:413-9.

11. Tobin-West $\mathrm{Cl}$, Kanu EN. Factors influencing the use of malaria prevention methods among women of reproductive age in peri-urban communities of Port harcourt city, Nigeria. Niger Postgrad Med J. 2016;23:6-11.

12. Uganda National Malaria Control Division (NMCD), Uganda Bureau of Statistics (UBOS), ICF. Uganda Malaria Indicator Survey 2018-19. Kampala, Uganda, and Rockville, Maryland, USA: NMCD, UBOS, and ICF; 2020.

13. Larsen K, Petersen JH, Budtz-Jørgensen E, Endahl L. Interpreting parameters in the logistic regression model with random effects. Biometrics. 2000;56:909-14.

14. Larsen K, Merlo J. Appropriate assessment of neighborhood effects on individual health: integrating random and fixed effects in multilevel logistic regression. Am J Epidemiol. 2005;161:81-8.

15. Tu Y-K, Kellett M, Clerehugh V, Gilthorpe MS. Problems of correlations between explanatory variables in multiple regression analyses in the dental literature. Br Dental J. 2005;199:457-61.

16. Goldstein H. Multilevel statistical models. London: Hodder Arnold; 2003.
17. Rasbash J, Charlton C, Browne WJ, Healy M, Cameron B. MLwiN Version 2.36. Centre for Multilevel Modelling, University of Bristol. 2015.

18. Manu G, Boamah-Kaali EA, Febir LG, Ayipah E, Owusu-Agyei S, Asante KP. Low utilization of insecticide-treated bed net among pregnant women in the middle belt of Ghana. Malar Res Treat. 2017;2017:7481210.

19. Hill J, Hoyt J, van Eijk AM, D'Mello-Guyett L, ter Kuile FO, Steketee R, et al. Factors affecting the delivery, access, and use of interventions to prevent malaria in pregnancy in sub-Saharan Africa: a systematic review and meta-analysis. PLoS Med. 2013;10:e1001488.

20. Pulford J, Hetzel MW, Bryant M, Siba PM, Mueller I. Reported reasons for not using a mosquito net when one is available: a review of the published literature. Malar J. 2011;10:83.

21. Krezanoski PJ, Nambogo N, Campbell JI, Bangsberg DR. Maternal attitudes about objectively monitored bednet use in rural Uganda. Malar Res Treat. 2016;2016:8727131.

22. Ricotta E, Oppong S, Yukich JO, Briët OJ. Determinants of bed net use conditional on access in population surveys in Ghana. Malar J. 2019;18:63.

23. Ruyange MM, Condo J, Karema C, Binagwaho A, Rukundo A, Muyirukazi Y. Factors associated with the non-use of insecticide-treated nets in Rwandan children. Malar J. 2016;15:355.

24. Adedokun ST, Uthman OA. Individual and contextual correlates of mosquito net use among women in Nigeria. Malar J. 2020;19:138.

25. WHO. Malaria: entomology and vector control. Geneva, World Health Organization; 2020. https://www.who.int/malaria/areas/vector_control/ en/. Accessed 8 June 2020.

26. Kanyangarara M, Hamapumbu H, Mamini E, Lupiya J, Stevenson JC, Mharakurwa S, et al. Malaria knowledge and bed net use in three transmission settings in southern Africa. Malar J. 2018;17:41.

27. Strecher VJ, Rosenstock IM. The health belief model: Cambridge handbook of psychology, health medicine; 1997, p. 117.

28. Kimbi HK, Nkesa SB, Ndamukong-Nyanga JL, Sumbele IU, Atashili J, Atanga MB. Knowledge and perceptions towards malaria prevention among vulnerable groups in the Buea Health District, Cameroon. BMC Public Health. 2014;14:883.

29. Lover AA, Sutton BA, Asy AJ, Wilder-Smith A. An exploratory study of treated-bed nets in Timor-Leste: patterns of intended and alternative usage. Malar J. 2011;10:199.

30. BBC Monitoring. Media Guide Uganda 2016. https://monitoring.bbc. co.uk/country/UGA. Accessed 8 June 2020.

31. Sangare LR, Weiss NS, Brentlinger PE, Richardson BA, Staedke SG, Kiwuwa $M S$, et al. Determinants of use of insecticide treated nets for the prevention of malaria in pregnancy: Jinja, Uganda. PLoS ONE. 2012;7:e39712.

32. Nkoka O, Chuang T-W, Chuang K-Y, Chen Y-H. Factors associated with insecticide-treated net usage among women of childbearing age in Malawi: a multilevel analysis. Malar J. 2018;17:372.

33. Njumkeng C, Apinjoh TO, Anchang-Kimbi JK, Amin ET, Tanue EA, Njua-Yafi C, et al. Coverage and usage of insecticide treated nets (ITNs) within households: associated factors and effect on the prevalance of malaria parasitemia in the Mount Cameroon area. BMC Public Health. 2019;19:1216.

34. Bennett A, Smith SJ, Yambasu S, Jambai A, Alemu W, Kabano A, et al. Household possession and use of insecticide-treated mosquito nets in Sierra Leone 6 months after a national mass-distribution campaign. PLoS ONE. 2012;7:e37927.

\section{Publisher's Note}

Springer Nature remains neutral with regard to jurisdictional claims in published maps and institutional affiliations. 